Received: December 2, 2019

Accepted: January 10, 2020

\section{Correspondence}

Filippo Raggini

Department of Orthopedics and Traumatology,

University Vita-Salute San Raffaele, via Olgettina 58,

20132 Milan, Italy

E-mail: filippo.raggini@gmail.com

Conflict of interest

The Authors declare no conflict of interest

How to cite this article: Raggini F, Placella G, Salini V. Subtrochanteric femur fractures: management and outcomes. Lo Scalpello 2020;34:525. https://doi.org/10.36149/0390-5276-008

(C) Ortopedici Traumatologi Ospedalieri d'Italia (O.T.O.D.i.) 2020

\section{(c) (i) () $\Theta$}

This is an open access article distributed in accordance with the CC-BY-NC-ND (Creative Commons Attribution-NonCommercial-NoDerivatives 4.0 International) license. The article can be used by giving appropriate credit and mentioning the license, but only for non-commercial purposes and only in the original version. For further information: https://creativecommons.org/licenses/by-nc-nd/4.0/deed.en

\title{
Subtrochanteric femur fractures: management and outcomes
}

\author{
Filippo Raggini', Giacomo Placella², Vincenzo Salini ${ }^{3}$ \\ ${ }^{1}$ Department of Orthopedics and Traumatology, University Vita-Salute San Raffaele, \\ Milan, Italy; ${ }^{2}$ Department of Orthopedics and Traumatology, San Raffaele Hospital \\ Scientific Institute, Milan, Italy; ${ }^{3}$ Department of Orthopedics and Traumatology, San \\ Raffaele Hospital Scientific Institute, Milan, Italy
}

\begin{abstract}
SUMMARY
Nowadays, subtrochanteric femur fractures (SFF) represent an important orthopedic problem to treat due to instability of the fragments, malunion and mechanical complications. The causes of failure in SFF include the lack of biomechanical consideration and the preoperative planning, wrong choice of synthesis, and reduction of the fracture or the surgical technique can compromise the correct post-operative recovery and the correct bone healing. It is important to understand the forces of this anatomical area in order to use right surgical approach. The following article provides a review of the current literature in order to outline epidemiology, treatment, outcomes and possible complications of subtrochanteric femur fractures.
\end{abstract}

Key words: subtrochanteric, complications, fixation, intramedullary nailing, plates

\section{Introduction}

SFF belong to extracapsular fracture of the femur. While in intracapsular fractures, the main problem is related to vascularization and lack of periosteum of the femoral neck, by contrast, in the extracapsular ones, the problem is mechanical and related to load-bearing ${ }^{~}$. SFF account for approximately 10 to $30 \%$ of all hip fractures ${ }^{2}$. Young people involved in high-energy traumas present complex fracture pattern, whereas old patients, generally females, present spiral fractures ${ }^{3}$. In addition to the now known risk factors for SFF such as metabolic bone disorders (e.g. bisphosphonates, diabetes etc.), the most important specific risk factor for delayed fracture healing in the SFF is mechanical instability ${ }^{4}$.

\section{Anatomy and classification}

There are different theories used to describe the anatomical area, but the most accredited definition is that of Fielding ${ }^{3}$ which identifies the interval between the lesser trochanter and around $5-7.5 \mathrm{~cm}$ below it, towards the femoral isthmus.

The lateral cortex is subjected to tensile forces, while the medial cortex is subjected to compressive forces. Due to tendon insertions, there is distraction and malrotation at the fracture site. The proximal fragment is flexed, abducted and externally rotated secondary to the pull of the iliopsoas, gluteus medius and short external rotators. Instead, the distal segment is adducted by the adductor magnus and longus tendon and this cause a varus deformity ${ }^{5}$. 
The X-rays of the entire femur should be obtained in order to study and classify the fracture. However, it is also critical to obtain X-rays of the hip and knee joints because of the association with a femoral shaft or distal femur fracture. CT or MRI can be performed in cases with equivocal plain radiographs to identify occult cortex lucencies ${ }^{6}$.

There are several classifications for SFF, but none of these guides treatment and establishes prognosis with satisfactory interobserver reproducibility ${ }^{7}$.

The Russel Taylor classification considers the presence or absence of the involvement of the piriformis fossa and the lesser trochanter. Today, it has lost its therapeutic and prognostic guidance, due to evolution of the implants used to repair $\mathrm{SFF}^{8}$. The Seinsheimer classification, instead, is more practical because it takes into consideration the segments of the fracture with the involvement both the medial and lateral cortices 9 .

Barbosa et al. states that there is still not a perfect classification system for treating SFF that determines a prognosis with satisfactory inter-observer reproducibility. The AOclassification considers the bone (femur = 3), the location (diaphysis $=2$ ), the energy of the trauma (A, B or C) and the mechanism (1, 2 or 3 ), but it includes the SFF in diaphyseal fractures which have a different mechanical and biological behavior. Among all, the last is the most widely used for its classification system that describes fracture morphology and mechanism ${ }^{8}$.

\section{Treatment and management}

Nowadays, different treatment methods are available for SFF. Non-surgical treatment of SFF, like traction, is still reserved for patients with extremely severe clinical comorbidities that contraindicate anesthetic and/or surgical procedures ${ }^{9}$.

Instead, the standard treatment for SFF is reduction and fixation with intra medullary nail (IMN) and clore reduction or extra medullary - open reduction and internal fixation (ORIF) with a plate (condylar blade plates, proximal femoral locking plates, percutaneous compression plates, less invasive stabilization systems, dynamic condylar screws) ${ }^{10}$.

But which is the best fixation method for SFF?

\section{IM nails}

Overall, IMN has become the gold standard for the treatment of SFF thanks to simple technique, rapid procedure and less invasivity. This device has different biomechanical advantages, such as greater strength, a shorter moment arm, a greater torsional stiffness and greater load-sharing capacity ${ }^{11}$.

It is, also, based on the concept of relative biologic fixation because it preserves vascularization at the fracture site, particularly the periosteal blood supply, and it promotes secondary bone healing by providing relative stability ${ }^{12}$.

It can decrease fixation failure, operative time and hospital length of stay compared to extramedullary devices ${ }^{13}$.

\section{Plates}

ORIF procedures can be performed with different types plate designs that can be chosen including the blade plate, the dynamic condylar plate or the proximal femoral locking plate. Although the IMN of SFF is the current treatment of choice, when the patient or fracture is not suitable for IMN submuscular plating is indicated. Such an instance might include an extension of the fracture into the greater trochanter or piriformis fossa, preventing a safe, adequate start site for a nail. A blade plate converts tensile force along the lateral cortex into a compressive force on the medial cortex, this construct requiring an intact medial cortex without comminution to avoid plate failure ${ }^{12}$.

\section{Complications and current evidence}

It is extremely important to choose the correct therapeutic approach because there are several post-operative complications following surgery for SFF. Even with modern implants, the complication rates in the treatment of the SFF remain high. Screw cut-out, femur fracture, varization, procurvatum malunion or nonunion, lack of consolidation and implant failure are the most common post-operative complications in $\mathrm{SFF}^{1}$.

In the SFF, screw cut-out is between 1.1 and $6.3 \%$ and represents $85 \%$ of fixation failures. The main causes of the cut-out are the instability of the fracture and, in particular, incorrect positioning of the lag screw which consists of the distance from the screw tip to the subchondral bone ${ }^{14}$. Thus, it is important for the surgeon to position the lag screw in the femoral head, in order to avoid serious implant failure due to screw cut-out ${ }^{15}$.

Studies show the diameter and the length of the nail play an important role in the surgery outcomes. Nails with a greater distal diameter lead to more frequent femoral shaft fractures than IMN nails with reduced distal diameter which also decline valgus offset ${ }^{16}$.

Jang et al. showed that short IMN (170 or $200 \mathrm{~mm}$ in length with 1 distal screw) may be used in a limited manner in high ST transverse fractures under the assumptions of anatomical reduction and fracture gap $\leq 1 \mathrm{~mm}$. Meanwhile, short CMN $200 \mathrm{~mm}$ in length with 2 distal screws may be a treatment option in most of ST transverse fractures regardless of the fracture level under the same set of assumptions ${ }^{17}$.

The incidence of implant failure in patients with SFF has been reported to be as high as $26 \%{ }^{18}$.

IMN implants with two cephalic blockage screws can determine Z-effect and reverse Z-effect as complications. These complications have been described as migrations of the cephalic screws in opposite directions due to factors such as low bone stock, excessively lateral entry point, varus reductions and severe medial comminution ${ }^{19}$.

Nevertheless, the proximal femur is largely composed of cortical bone and bears enormous stress varus ${ }^{20}$. Thus, SFF are quite prone to nonunion ${ }^{21}$. 
Subtrochanteric fracture nonunion is more challenging than the treatment of a fresh fracture because of bone loss, retained broken implants, loss of reduction, and the compromised osteogenic potential of local tissue ${ }^{22}$.

What is extremely important, before nailing, is to have an intact cortex and to reduce the fracture in order to identify the correct nail entry point (trochanteric or pyriformis fossa start site) ${ }^{23}$. No particular difference was found between trochanteric and pyriformis starting nails at the initial site of the fossa, even if with the latter, there is an anatomical alignment with the medullary canal and this can prevent the reduction of the valgus of the proximal fragment that can be caused, instead, by the design of trochanteric starting nails ${ }^{24}$.

Lack of medial cortical support is a well-known risk factor for subtrochanteric nonunion. The anatomical reduction restores the medial cortical support, if there is a fracture without comminution zone. In fact, intact medial cortical support counteracts flexion and torque forces during post-operative mobilization. However, in comminute fractures with no medial cortical support the IMN nail acts as a load sharing device. In this case, a comminution of posteromedial cortex is a relative indication for use of plating ${ }^{25}$.

On the other hand, with open plating, anatomical reduction and rigid fixation are reportedly associated with increased risk of nonunion, infection, implant failure, and re-operation due to extensive exposure, soft tissue stripping leading to devitalization of fragments, and loss of fracture hematoma ${ }^{26}$.

The conventional approach (open) with plates promotes important local devascularization and increases the rates of infection, pseudarthrosis, and osteosynthesis failure ${ }^{27}$.

Some authors have analyzed the use of plates with fixed angle screw to treat SFF.

Kuzyk et al. ${ }^{28}$ compared IMN to plates for SFF and reported better results for IMN implants in terms of operating time and lower rate of implant failure.

Wirtz et al. ${ }^{29}$, instead, demonstrated several complications with the open reduction technique and internal fixation with PF-LCP (Synthes, West Chester PA, USA, such as infection, cut-out, and varus collapse, requiring new surgical procedures. They emphasized that, contrary to intramedullary implants, PF-LCPs do not allow for fracture accommodation, which is critical for consolidation in fractures with loss of posteromedial support.

Jang et al. ${ }^{30}$ showed that biologic plating with minimally invasive surgery, which preserves blood supply around the fracture site, allows good surgical outcomes in patients with SFF.

Compared to fixed angle plating and proximal femoral locking plates, the most successful type of plating involves the use of 95 degree fixed angle blade plates ${ }^{31}$.

Finally, several authors declare that the quality of fracture reduction is much more important than the choice of the implant. In fact, in a well-reduced fracture, the literature demonstrates that the results of intra- and extra-medullary fixation using the biological technique (minimally invasive) are $\operatorname{similar}^{8}$.

\section{Conclusions}

Optimal management of SFF remains a challenge for the orthopedic surgeon. Early anatomical reduction and surgical fixation represents the best bet to reduce the risk of complications like non-union and avascular necrosis in treating SFF.

It is essential to study the radiographic image of the fracture, assess the integrity of the bone and adjacent soft tissues and carry out a correct pre-operative planning.

The prevention of restoration of the medial cortical support and post-operative varus malalignment are the most critical factors to prevent nonunion after IMN nailing of SFF.

If there is an intertrochanteric extension of the fracture, it is important to assess the integrity of the potential start site if intramedullary nailing is selected for fixation. The key point to reduce the risk of complications is the quality of the reduction which lowers the stress on the implant, increases the bone contact and makes the consolidation easier.

Careful soft tissue dissection, however, is deemed mandatory during open reduction.

For "simple" fractures, with medial cortical integrity and no comminuted fragments, plates with minimally invasive surgery or nails are both good options. Instead, trochanteric extensions may favor plating.

By contrast, the best choice for "complex" fractures, trasversal and comminuted ones, is to nail and if the surgeon prefers plating, it is better to use a strong plate with "biologic" techniques to preserve bone integrity.

A long IMN nail is always a safe option, but avoid malreduction. It has, also, been proven to have increased success rates in these fractures and should be preferred over extramedullary plate fixation systems.

Plating should be performed using less invasive techniques.

Different aspects still remain uncovered regarding the management of SFF. Therefore, further investigative studies and an evidence-based approach are needed in order to improve our knowledge of the subject and a consequent improvement in clinical results.

\section{References}

1 Carpintero P, Caeiro JR, Carpintero R, et al. Complications of hip fractures: a review. World J Orthop 2014;5:402-11.

2 Lee MA. Subtrochanteric hip fractures, 2010 (https://emedicine. medscape.com/article/1247329-overview).

3 Fielding JW. Subtochanteric fractures. Clin Orthop Relat Res 1973;92:86-99.

4 Panteli M, Mauffrey C, Giannoudis PV. Subtrochanteric fractures: issues and challenges. Injury 2017;48:2023-6. https://doi. org/10.1016/j.injury.2017.09.001

5 Joglekar SB, Lindvall EM, Martirosian A. Contemporary management of subtrochanteric fractures. Orthop Clin North Am 2015;46:21-35.

6 Feldman F. Atypical diaphyseal femoral fractures-new aspects. Skeletal Radiol 2012;41:75-81. https://doi.org/10.1007/s00256-011-1130-6 
7 Guyver PM, McCarthy MJ, Jain NP, et al. Is there any purpose in classifying subtrochanteric fractures? The reproducibility of four classification systems. Eur J Orthop Surg Traumatol 2014;24:513-8.

8 Barbosa de Toledo Lourenco PR, Pires RE. Subtrochanteric fractures of the femur: update. Rev Bras Ortop 2016;51:246-53.

9 Miedel R, Törnkvist H, Ponzer S, et al. Musculoskeletal function and quality of life in elderly patients after a subtrochanteric femoral fracture treated with a cephalomedullary nail. J Orthop Trauma 2011;25:208-13.

10 Forward DP, Doro CJ, O'Toole RV, et al. A biomechanical comparison of a locking plate, a nail, and a 95 degrees angled blade plate for fixation of subtrochanteric femoral fractures. $\mathbf{J}$ Orthop Trauma 2012;26:334-40. https://doi.org/10.1097/BOT. 0b013e3182254ea3

11 Johnson KD, Tencer AF, Sherman MC. Biomechanical factors affecting fracture stability and femoral bursting in closed intramedullary nailing of femoral shaft fractures, with illustrative case presentations. J Orthop Trauma 1987;1:1-11.

12 Santolini E, Goumenos SD, Giannoudi M, et al. Femoral and tibial blood supply: a trigger for non-union? Injury 2014;45:1665-73. https://doi.org/10.1016/j.injury.2014.09.006

13 Jackson C, Tanios M, Ebraheim N. Management of subtrochanteric femur fracturs: a review of tecent literature. Adv Orthop 2018;2018:1326701. https://doi.org/10.1155/2018/1326701

14 Lorich DG, Geller DS, Nielson JH. Osteoporotic pertrochanteric hip fractures: management and current controversies. Instr Course Lect 2004;53:441-54.

15 Kim WY, Han CH, Park JI, et al. Failure of intertrochanteric fracture fixation with a dynamic hip screw in relation to pre-operative fracture stability and osteoporosis. Int Orthop 2001;25:360-2.

16 Saudan M, Lübbeke A, Sadowski C, et al. Pertrochanteric fractures: is there an advantage to an intramedullary nail? A randomized, prospective study of 206 patients comparing the dynamic hip screw and proximal femoral nail. J Orthop Trauma 2002;16:386-93.

17 Jang CY, Bang SH, Kim WH, et al. Effect of fracture levels on the strength of bone-implant constructs in subtrochanteric fracture models fixed using short cephalomedullary nails: a finite element analysis. Injury 2019;50:1883-8. https://doi.org/10.1016/j.injury.2019.08.014

18 Fielding JW, Cochran GV, Zickel RE. Biomechanical characteristics and surgical management of subtrochanteric fractures. Orthop Clin North Am 1974; 5:629-50.
19 Pires RE, Santana EOJr, Santos LE, et al. Failure of fixation of trochanteric femur fractures: clinical recommendations for avoiding Z-effect and reverse Z-effect type complications. Patient Saf Surg 2011;5:17.

20 Melis GC, Chiarolini B, Tolu S. Surgical treatment of subtrochanteric fractures of the femur: biomechanical aspects. Ital J Orthop Traumatol 1979;5:163-86.

21 Giannoudis PV, Ahmad MA, Mineo GV, et al. Subtrochanteric fracture non-unions with implant failure managed with the "Diamond" concept. Injury 2013;44:S76-S81. https://doi.org/10.1016/ S0020-1383(13)70017-2.

22 Charnley GJ, Ward AJ. Reconstruction femoral nailing for nonunion of subtrochanteric fracture: a revision technique following dynamic condylar screw failure. Int Orthop 1996;20:55-7.

23 Ostrum RF, Marcantonio A, Marburger R. A critical analysis of the eccentric starting point for trochanteric intramedullary femoral nailing. J Orthop Trauma 2005;19:681-6. https://doi. org/10.1097/01.bot.0000184145.75201.1b

24 Kelly-Petterson P, Samuelsson B, Muren O, et al. Waiting time to surgery is correlated with an increased risk of serious adverse events during hospital stay in patients with hip-fracture: a cohort study. Int J Nurs Stud 2017; 69:91-7.

25 Celebi L, Can M, Muratli HH, et al. Indirect reduction and biological internal fixation of comminuted subtrochanteric fractures of the femur. Injury 2006;37:740-50. https://doi.org/10.1016/j. injury.2005.12.022

26 Asher MA, Tippett JW, Rockwood CA, et al. Compression fixation of subtrochanteric fractures. Clin Orthop Relat Res 1976;117:202-8

27 Saini P, Kumar R, Shekhawat V, et al. Biological fixation of comminuted subtrochanteric fractures with proximal femur locking compression plate. Injury 2013;44:226-31.

28 Kuzyk PR, Paul RT, Bhandari M. Intramedullary versus extramedullary fixation for subtrochanteric femur fractures. J Orthop Trauma 2009;23:465-70.

29 Wirtz C, Abbassi F, Evangelopoulos DS, et al. High failure rate of trochanteric fracture osteosynthesis with proximal femoral locking compression plate. Injury 2013;44:751-6.

30 Jang JH, Ahn JM, Lee HJ, et al. Surgical outcomes of biologic fixation for subtrochanteric fracture using locking compression plates. Hip Pelvis 2017;29:68-76.

31 Koval KJ, Rezaie N, Yoon RS. In: Proximal femur fractures. Cham: Springer 2018, pp. 101-12. 